International Mathematical Forum, 1, 2006, no. 24, 1145 - 1151

\title{
The Characterization of the Matrix Sequences on the Sequence Space $b v_{p}$
}

\author{
Hasan Furkan \\ Kahramanmaraş Sütçü İmam Üniversitesi \\ Fen Edebiyat Fakültesi \\ Matematik Bölümü \\ 46100 Kahramanmaraş, Turkey \\ e-mail:hasanfurkan@hotmail.com
}

\begin{abstract}
In [7] Khan and Riazuddin characterized the class $(b v: C)$ of matrix sequences and Başar, Altay and Mursaleen [3] characterized the class $\left(b v_{p}: c\right)$ of infinite matrices. The main purpose of the present paper is to determine the necessary and sufficient conditions on a matrix sequence $\mathcal{A}=\left(A_{v}\right)$ in order that $\mathcal{A}$ belongs to the matrix class $\left(b v_{p}: C\right)$, where $1<p \leq \infty$.
\end{abstract}

Mathematics Subject Classification: $40 \mathrm{C} 05$.

Keywords: Matrix sequences, double sequence and matrix sequence transformation.

\section{Preliminaries, Background and Notation}

By $w$, we denote the space of all real valued sequences. Any vector subspace of $w$ is called a sequence space. We write $\ell_{\infty}, c, c_{0}$ and $b v$ for the spaces of all bounded, convergent, null and bounded variation sequences, respectively. Also by $b s, c s, \ell_{1}$ and $\ell_{p}$, we denote the spaces of all bounded, convergent, absolutely and $p$-absolutely convergent series, respectively. We also denote by $C$ and $C_{0}$, the spaces of all convergent and null double sequences, respectively.

For the sequence spaces $\lambda$ and $\mu$ define the set $S(\lambda, \mu)$ by

$$
S(\lambda, \mu)=\left\{z=\left(z_{k}\right) \in w: x z=\left(x_{k} z_{k}\right) \in \mu \text { for all } x \in \lambda\right\} .
$$

With the notation of (1), $\alpha$-, $\beta$ - and $\gamma$ - duals of a sequence space $\lambda$, which are respectively denoted by $\lambda^{\alpha}, \lambda^{\beta}$ and $\lambda^{\gamma}$, are defined by

$$
\lambda^{\alpha}=S\left(\lambda, \ell_{1}\right), \quad \lambda^{\beta}=S(\lambda, c s), \quad \lambda^{\gamma}=S(\lambda, b s) .
$$


A sequence $x=\left(x_{k}\right)$ is almost convergent to $L$ if and only if

$$
\lim _{n \rightarrow \infty} \frac{x_{v}+x_{v+1}+\cdots+x_{v+n-1}}{n}=L \quad \text { uniformly in } v=1,2,3, \ldots
$$

Let $\lambda, \mu$ be two sequence spaces and let $A=\left(a_{n k}\right)$ be an infinite matrix of real or complex numbers $a_{n k}$, where $n, k \in \mathbb{N}$. Then, the matrix $A$ defines $A$ - transformation from $\lambda$ into $\mu$, if for every sequence $x=\left(x_{k}\right) \in \lambda$ the sequence $A x=\left((A x)_{n}\right)$, the $A$-transform of $x$, exists and is in $\mu$; where $(A x)_{n}=$ $\sum_{k} a_{n k} x_{k}$. For simplicity in notation, here and in what follows, the summation without limits runs from 0 to $\infty$. By $(\lambda: \mu)$, we denote the class of all such matrices. A sequence $x$ is said to be $A$-summable to $l$ if $A x$ converges to $l$ which is called as the $A$-limit of $x$.

For a sequence space $\lambda$, the matrix domain $\lambda_{A}$ of an infinite matrix $A$ is defined by

$$
\lambda_{A}=\left\{x=\left(x_{k}\right) \in w: A x \in \lambda\right\}
$$

The idea of $\mathcal{A}$-summability, explained below, was introduced by H. T. Bell in his doctoral work [5]. For $v=1,2,3, \ldots$, let $A_{v}=\left(a_{n k}(v)\right)$ be a infinite matrix of real numbers and let $\mathcal{A}$ be a sequence of matrices $\left(A_{v}\right)$ and $\lambda \subset w, \mu \subset W$. Then, the matrix sequences $\mathcal{A}=\left(A_{v}\right)$ defines a transformation from $\lambda$ into $\mu$ if for every sequence $\left(x_{k}\right) \in \lambda$ the double sequence $\mathcal{A} x=\left((\mathcal{A} x)_{n}^{v}\right)_{n, v=0}^{\infty}$, the $\mathcal{A}$ transform of $x$ exists and is in $\mu$; where $(\mathcal{A} x)_{n}^{v}=\sum_{k} a_{n k}(v) x_{k}$. For simplicity in notation, here and after we abbreviate the infinite sum notation $\sum_{k=0}^{\infty}$ as $\sum_{k}$. By $(\lambda: \mu)$, we denote the class of all such matrix sequences. A sequence $x$ is said to be $\mathcal{A}$-summable to $L$ if $\lim _{n}(\mathcal{A} x)_{n}^{v}=L$ uniformly in $v$. We shall write throughout for brevity that

$$
\begin{array}{r}
\tilde{a}_{n k}(v)=\sum_{j=k}^{\infty} a_{n j}(v), \\
a(n, k, v)=\sum_{i=1}^{n} a_{i k}(v),
\end{array}
$$

for all $n, k, v \in \mathbb{N}$. If we choose $\mathcal{A}=\left(a_{n k}(v)\right)$ as

$$
a_{n k}(v)=\left\{\begin{array}{ccc}
\frac{1}{n+1} & , & (v \leq k \leq v+n) \\
0 & , & \text { (otherwise) }
\end{array}\right.
$$

in [5], although no ordinary limitation method can correspond to almost convergence defined in[8], it is shown that this is possible using matrix sequences. 
Moreover, in the cases of $a_{n k}(v)=a_{n k}$ and $a_{n k}(v)=\delta_{n k}$, the Kronecker delta, for all $n, k, v \in \mathbb{N}$; the method $\mathcal{A}$ respectively corresponds to the usual summability method $A$ and the identity method $I$ which is equivalent to the ordinary convergence. If

$$
a_{n k}(v)=\frac{1}{n+1} \sum_{j=0}^{n} a_{v+j, k} ; \quad(k, n, v \in \mathbb{N})
$$

then the method $\mathcal{A}$ corresponds to almost summability method given in [9]. Again, recently, in [6], $\mathcal{A}=\left(a_{n k}(v)\right)$ is chosen to be

$$
a_{n k}(v)=\left\{\begin{array}{ccc}
\frac{1}{n+1} & , & \left(k=\sigma^{j}(v), 0 \leq j \leq k\right) \\
0, & \text { (otherwise) }
\end{array}\right.
$$

and it is shown that it corresponds to $\sigma$-convergence. Therefore, the method $\mathcal{A}$ is more general and more comprehensive than the ordinary limitation method A.

\section{Main Results}

In this section, the space $b v_{p}$ of sequences of $p$-bounded variation was defined and studied by Başar and Altay in [2], where

$$
b v_{p}=\left\{x=\left(x_{k}\right) \in w: \sum_{k}\left|x_{k}-x_{k-1}\right|^{p}<\infty\right\},(1 \leq p<\infty) .
$$

It was proved that $b v_{p}$ is a $B K$-space which is linearly isomorphic to the space $\ell_{p}$ and the inclusion $b v_{p} \supset \ell_{p}$ strictly holds. The $\alpha-, \beta$ - and $\gamma$-duals of the space $b v_{p}$ are determined together with the fact that $b v_{2}$ is the only Hilbert space among the spaces $b v_{p}$. The continuous dual of the space $b v_{p}$ is determined and is given in the following lemma which is required in the proof of our next theorem:

Lemma 2.1 [1, Theorem 3.2] Define the spaces $d_{1}$ and $d_{q}$ consisting of all sequences $a=\left(a_{k}\right)$ normed by

$$
\|a\|_{d_{1}}=\sup _{k, n \in \mathbb{N}}\left|\sum_{j=k}^{n} a_{j}\right|<\infty
$$

and

$$
\|a\|_{d_{q}}=\left(\sup _{n \in \mathbb{N}} \sum_{k}\left|\sum_{j=k}^{n} a_{j}\right|^{p}\right)^{1 / q}<\infty, \quad(1<q<\infty) .
$$

Then, $\left\{b v_{1}\right\}^{*}$ and $\left\{b v_{p}\right\}^{*}$ are isometrically isomorphic to $d_{1}$ and $d_{q}$, respectively. 
The basis of the space $b v_{p}$ is also constructed and given by the following lemma:

Lemma 2.2 [2, Theorem 4.1] Define the sequence $b^{(k)}=\left\{b_{n}^{(k)}\right\}_{n \in \mathbb{N}}$ of the elements of the space $b v_{p}$ for every fixed $k \in \mathbb{N}$ by

$$
b_{n}^{(k)}=\left\{\begin{array}{ll}
0, & (n<k) \\
1, & (n \geq k))
\end{array} ;(k, n \in \mathbb{N})\right.
$$

Then the sequence $\left\{b_{n}^{(k)}\right\}_{n \in \mathbb{N}}$ is a basis for the space $b v_{p}$ and any $x \in b v_{p}$ has a unique representation of the form

$$
x=\sum_{k} \lambda_{k} b^{(k)}
$$

where $\lambda_{k}=x_{k}-x_{k-1}$ for all $k \in \mathbb{N}$.

More recently, the paranormed sequence spaces $b v(u, p)$ and $b v_{\infty}(u, p)$ have been introduced and $\alpha-, \beta-, \gamma$-duals, the Schauder basis and the characterizations of related matrix mappings have been given by Başar, Altay and Mursaleen in [3].

The main purpose of this paper is to characterize the class $\left(b v_{p}: C\right)$. Before giving the main theorem, we state the following lemma given by Başar and Altay [2]:

Lemma 2.3 [2, Theorem 2.2] The space bv of sequences of p-bounded variation is linearly isomorphic to the space $\ell_{p}$, i.e., b $v_{p} \cong \ell_{p}$; where $1 \leq p \leq \infty$.

Theorem 2.4 Let $1<p<\infty$. Then $\mathcal{A} \in\left(b v_{p}: C\right)$ if and only if

$$
\begin{gathered}
\sup _{m, v \in \mathbb{N}} \sum_{k}\left|\sum_{j=k}^{m} a_{n j}(v)\right|^{q}<\infty ; \quad(n \in \mathbb{N}) \\
\sup _{n, v \in \mathbb{N}} \sum_{k}\left|\tilde{a}_{n k}(v)\right|^{q}<\infty, \\
\left.\lim _{n} \tilde{a}_{n k}(v)=\alpha_{k} \text { (uniformly in } v\right) .
\end{gathered}
$$

Proof: Let $\mathcal{A} \in\left(b v_{p}: C\right)$ and $1<p<\infty$. Then $\mathcal{A} x$ exists for every $x \in b v_{p}$ and this implies that $\left\{a_{n k}(v)\right\}_{k, v \in \mathbb{N}} \in b v_{p}^{\beta}$ for each $n, v \in \mathbb{N}$ which shows the necessity of (5).

Let us consider the following relation between the sequences $x=\left(x_{k}\right)$ and $y=\left(y_{k}\right)$ :

$$
y_{k}=(\Delta x)_{k}= \begin{cases}x_{0}, & k=0 \\ x_{k}-x_{k-1}, & k \geq 1\end{cases}
$$


By applying the Abel's partial sum to the $m^{\text {th }}$ partial sum of the series $\sum_{k} a_{n k}(v) x_{k}$ and letting $m \rightarrow \infty$, one can see that

$$
\begin{aligned}
\sum_{k} a_{n k}(v) x_{k} & =\sum_{k} a_{n k}(v)\left(\sum_{j=0}^{k} \Delta x_{j}\right) \\
& =\sum_{j} \sum_{k=j}^{\infty} a_{n k}(v) \Delta x_{j}=\sum_{j} \tilde{a}_{n k}(v) y_{j} ; \quad(n, v \in \mathbb{N}) .
\end{aligned}
$$

That is, we obtain

$$
\sum_{k} a_{n k}(v) x_{k}=\sum_{j} \tilde{a}_{n k}(v) y_{k}
$$

Taking supremum over $n, v$ and applying Hölder's inequality we obtain from (9) that

$$
\sup _{n \in \mathbb{N}} \sum_{k}\left|a_{n k}(v) x_{k}\right| \leq \sup _{n \in \mathbb{N}}\left(\sum_{k}\left|\tilde{a}_{n k}(v)\right|^{q}\right)^{1 / q}\left(\sum_{k}\left|y_{k}\right|^{p}\right)^{1 / p}<\infty .
$$

This implies the necessity of (6).

To prove the necessity of (7) we define, for every fixed $k \in \mathbb{N}$, the sequence of the elements of $b v_{p}$ as

$$
b_{n}^{(k)}= \begin{cases}0, & (n<k) \\ 1, & (n \geq k)\end{cases}
$$

Because of the $\mathcal{A}$-transform of every $x \in b v_{p}$ exists and lies in $C$ by the hypothesis, $\mathcal{A} b_{n}^{(k)}=\left\{\tilde{a}_{n k}(v)\right\}_{n \in \mathbb{N}}$ is also in $C$ for every fixed $k \in \mathbb{N}$, which shows the necessity of $(7)$.

Conversely suppose that the conditions (5)-(7) hold, and take any $x=\left(x_{k}\right)$ in the space $b v_{p}$. Then, $\mathcal{A} x$ exists. We observe for every $m, n \in \mathbb{N}$ that

$$
\sum_{j=0}^{m}\left|\sum_{k=j}^{m} a_{i j}(v) x_{j}\right| \leq \sup _{i, v \in \mathbb{N}} \sum_{j}\left|\tilde{a}_{i j}(v) y_{j}\right|
$$

which leads us to the following fact, by letting $m, n \rightarrow \infty$ in (7) and using (5),

$$
\sum_{j}\left|\sum_{k=j}^{\infty} \alpha_{j}\right|<\infty
$$

Hence, $\left(\alpha_{k}\right) \in b v_{p}$ which implies that the series $\sum \alpha_{k} x_{k}$ is convergent for every $x \in b v_{p}$. 
Let us now consider the equality obtained from (9) with $a_{n k}(v)-\alpha_{k}$ instead of $a_{n k}(v)$ :

$$
\sum_{k}\left[a_{n k}(v)-\alpha_{k}\right] x_{k}=\sum_{k} b_{n k}(v) y_{k}, \quad(n, v \in \mathbb{N}) ;
$$

where $\mathcal{B}=\left(b_{n k}(v)\right)$ with $b_{n k}(v)=\sum_{j=k}^{\infty} a_{n j}(v)-\alpha_{k}$ for all $n, k, v \in \mathbb{N}$. Therefore we have at this stage from [10] with $\alpha_{k}=0$ for all $k \in \mathbb{N}$ that the matrix $\mathcal{B}$ belongs to the class $\left(\ell_{p}: C_{0}\right)$. Thus we see by $(12)$ that

$$
\lim _{n \rightarrow \infty} \sum_{k}\left(a_{n k}(v)-\alpha_{k}\right) x_{k}=0
$$

which means that $\mathcal{A} x \in C$ whenever $x \in b v_{p}$ and this is what we wished to prove.

For the case $p=\infty$ the conditions for $\mathcal{A} x \in\left(b v_{\infty}, C\right)$ are (6), (7) and

$$
\sum_{k}\left|\sum_{j=k}^{\infty} a_{n j}(v)\right|<\infty
$$

The proof is similar to the above proof.

It is trivial that the sequence spaces $b v(u, p)$ and $b v_{\infty}(u, p)$ may be reduced to $b v_{p}$ and $b v_{\infty}$ in the case $p_{k}=p$ and $u_{k}=1(k=0,1,2, \ldots)$ respectively. If we take $p=1$ then Theorem 2.4 reduces to Theorem 2.1 of [7]. In the case $p_{k}=p$ and $u_{k}=1(k=0,1,2, \ldots)$ if we take $\mathcal{A}=A$ for every $v$ then Theorem 2.4 reduces to Theorem 4.2 of [3]. Hence, Theorem 2.4 is more general than the corresponding results of [7] and [3].

\section{References}

[1] A. M. Akhmedov and F. Başar, On the fine spectra of the difference operator $\Delta$ over the sequence space $b v_{p},(1 \leq p<\infty)$, Acta Math. Sin. Eng. Ser. (to appear).

[2] F. Başar and B. Altay, On the space of sequences p- bounded variation and related matrix mappings, Ukrainian Math. J. 55(1)(2003), 136-147.

[3] F. Başar, B. Altay and M. Mursaleen, Some generalizations of the space $b v_{p}$ of $p$-bounded variation sequences, (under cominication).

[4] F. Başar, Matrix sequences and certain sequence spaces of double sequences, Soochow J. Math. 23(1997), no. 2, 213-226. 
[5] H. T. Bell, a-Summability, PhD. thesis, Lehigh University, 1971.

[6] H. Çoşkun and C. Çakan, On some new inequalities related to $F_{\mathcal{B}^{-}}$ convergence, Tamsui Oxf. J. Math. Sci. 19(2)(2003), 131-140.

[7] F. M. Khan and M. Riazuddin, Matrix transformation of sequnces of bounded variation, Matematigki Vesnik 3(16)(31),(1979), 287-293.

[8] G. G. Lorentz, A contribution to the theory of divergent sequences, Acta Math. 80(1948), 167-190.

[9] J. P. King, Almost summable sequences, Proc. Amer. Math. Soc. 17(1966), 1219-1225.

[10] E. Savaş, Matrix transformations of some generalized sequence spaces, J. Orissa. Math. Soc., 4(1)(1985), 37-51.

Received: October 21, 2005 